\title{
NLTE wind models of A supergiants
}

\author{
Jiří Krtička ${ }^{1}$ and Jiří Kubát ${ }^{2}$ \\ ${ }^{1}$ Institute of Theoretical Physics and Astrophysics, Masaryk University, CZ-611 37 Brno, \\ Czech Republic, email: krticka@physics.muni.cz \\ ${ }^{2}$ Astronomical Institute, Academy of Sciences of the Czech Republic, CZ-251 65 Ondřejov, \\ Czech Republic, email: kubat@sunstel.asu.cas.cz
}

\begin{abstract}
We present new numerical models of line-driven stellar winds of A supergiants. Statistical equilibrium (NLTE) equations of the most abundant elements are solved, and properly obtained occupation numbers are used to calculate consistent radiative force and radiative heating terms. Wind density, velocity, and temperature are calculated as solutions of the model's hydrodynamical equations. Our models allow for the calculation of the wind mass-loss rate and terminal velocity.
\end{abstract}

Keywords. Stars: atmospheres, stars: early-type, stars: winds, outflows, radiative transfer

\section{Introduction}

The stellar winds of A supergiants have properties similar to those of their hotter counterparts, i.e., the stellar winds of OB supergiants. These winds are accelerated by the absorption of radiation mainly in resonance lines of elements such as carbon, nitrogen, oxygen, and iron. However, the domain of A supergiant winds seems to be overlooked both by the wind theorists and by the observers. Reliable data on wind mass-loss rates and terminal velocities are scarce and there is a lack of detailed wind models. To fill this gap we present our NLTE wind models which are capable of predicting wind parameters (i.e., mass-loss rates and terminal velocities) of A supergiants.

\section{Model assumptions}

The preceding version of our wind code is described in detail by Krtička \& Kubát (2004). Here we only summarise basic model properties and describe improvements with respect to the published version.

The basic model assumptions are the following:

- We assume a stationary spherically-symmetric flow.

- We solve the continuity equation, the momentum equation and the energy equation for each component of the flow, namely for absorbing ions, nonabsorbing ions (hydrogen and helium), and electrons. Nonabsorbing ions are ions for which the effect of a radiative force may be neglected (e.g., hydrogen and helium if Population I/II stars are considered). The possibility of helium decoupling is not accounted for (cf. Krtička \& Kubát 2005).

- Level occupation numbers of model ions are obtained from NLTE rate equations with the inclusion of a superlevel concept (Anderson 1989).

- Radiation transfer is split into two parts. Line radiative transfer is solved in the Sobolev approximation (Rybicki \& Hummer 1978) neglecting continuum opacity sources, line overlaps, and multiple scattering. Continuum radiative transfer is formally solved using the Feautrier method by including all free-free and bound-free transitions of the model ions, however neglecting line transitions. Thus, we neglect line-blocking. 


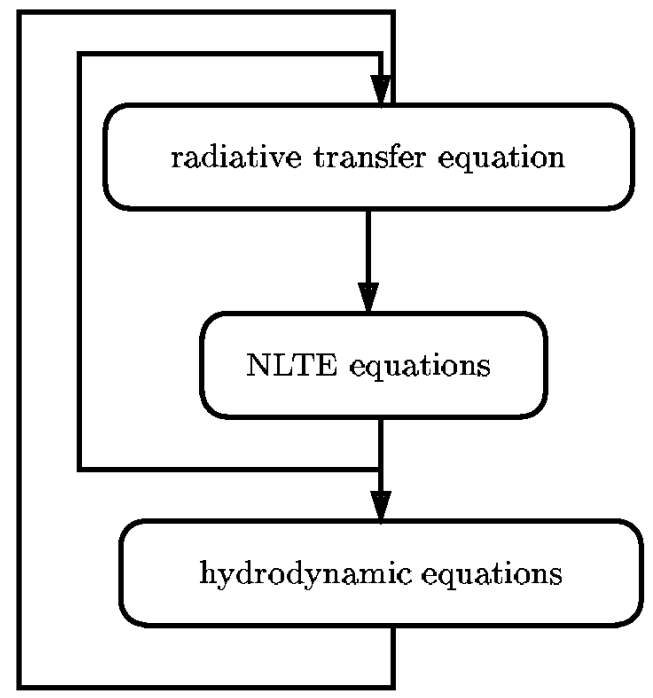

Figure 1. The scheme of the model calculation procedure. Note that the outer iteration cycle which determines the correct value of the mass-loss rate to fulfil the critical condition is not displayed here.

- Derived occupation numbers are used to calculate the radiative force in the hydrodynamic equations.

- The radiative cooling/heating term is obtained by adding the contributions from all free-free, bound-free, and collisional bound-bound transitions of the model ions. For the calculation of this term we use the thermal balance of electrons method (Kubát et al. 1999).

- The description of the transition region between the quasi-static stellar atmosphere and the supersonic stellar wind is simplified. This region is important for continuum formation (Pauldrach 1987). To obtain the correct ionizing flux we included this subsonic region in our models, i.e., the boundary velocity at the stellar surface is set to some low value well below the sonic speed.

- The calculation of models below and above the critical point is split into two parts. Thus we neglect the influence of the radiation emitted in the region above the critical point on the region below the critical point.

- We neglect Gayley-Owocki heating (Gayley \& Owocki 1994) which is not important for stars considered in this paper (see Krtička \& Kubát 2001).

- We also neglect X-ray radiation which may influence the ionization balance.

\section{Model description}

The basic procedure for the wind model calculation is displayed in Figure 1. In the inner iteration cycle statistical equilibrium equations together with the radiative transfer equation are solved. Calculated level occupation numbers and mean radiative intensities are used to solve the hydrodynamic equations. Since the derived hydrodynamic structure influences the occupation numbers, another iteration cycle is necessary to account for this coupling. An additional (outer) iteration cycle, which is not displayed in the Figure 1, is used to fulfil the critical condition. During this outer iteration cycle the correct value of the wind mass-loss rate is determined. 


\subsection{Continuum radiative transfer}

In the radiative transfer equation in continuum we neglect wind motion and bound-bound opacity sources. Thus, the radiative transfer equation in the spherical coordinates has the form

$$
\mu \frac{\partial I(r, \nu, \mu)}{\partial r}+\frac{1-\mu^{2}}{r} \frac{\partial I(r, \nu, \mu)}{\partial \mu}=\eta(r, \nu, \mu)-\chi(r, \nu, \mu) I(r, \nu, \mu),
$$

where $I(r, \nu, \mu)$ is the specific intensity of radiation, $\mu=\cos \theta$ is the direction cosine, $\nu$ is the frequency and $\chi(r, \nu, \mu)$ and $\eta(r, \nu, \mu)$ are the emission and absorption coefficients, respectively. The solution of this equation is obtained using the Feautrier (1964) method (for a description, see Mihalas \& Hummer (1974) or Kubát (1993)). The resulting tridiagonal system of equations is solved using the numerical package LAPACK (Anderson et al. 1999) (http://www.cs.colorado.edu/ lapack) instead of the classical Gaussian scheme. All bound-free transitions of explicit ions, free-free transitions of hydrogen and helium and light scattering due to free electrons contribute to the source function. The radiative transfer equation is solved only for frequencies above the hydrogen ionization edge. For frequencies lower than the hydrogen ionization limit we neglect the radiation-matter interaction and use an approximation $J_{\nu}(r)=4 W(r) H_{c}$, where $W(r)=\frac{1}{2}\left(1-\mu_{*}\right)$ is the dilution factor.

The lower boundary flux is taken either from model atmospheres of Kubát (2003) or of Hubeny \& Lanz (1995).

\subsection{Line radiative transfer}

The line radiative transfer is solved in the Sobolev approximation with the neglect of continuum radiation as (Castor 1974, Rybicki \& Hummer 1978)

$$
\bar{J}_{i j}=(1-\beta) S_{i j}+\beta_{c} I_{c}
$$

where $\bar{J}_{i j}=\int_{0}^{\infty} \mathrm{d} \nu \int_{-1}^{1} \mathrm{~d} \mu \phi_{i j}(\nu) I(r, \nu, \mu)$ is the mean intensity, $\phi_{i j}(\nu)$ is the line profile, $I_{c}$ is the specific intensity emerging from the star, $\beta=\frac{1}{2} \int_{-1}^{1} \mathrm{~d} \mu \frac{1-e^{-\tau_{\mu}}}{\tau_{\mu}}, \beta_{c}=$ $\frac{1}{2} \int_{\mu_{*}}^{1} \mathrm{~d} \mu \frac{1-e^{-\tau \mu}}{\tau_{\mu}}, \mu_{*}=\left(1-R_{*}^{2} / r^{2}\right)^{1 / 2}$, and the source function $S_{i j}=\eta_{i j} / \chi_{i j}$.

\subsection{Statistical equilibrium equations}

Occupation numbers $N_{i}$ of atoms in the state $i$ are given by the solution of static statistical equilibrium (NLTE) equations

$$
\sum_{j \neq i} N_{j} P_{j i}-N_{i} \sum_{j \neq i} P_{i j}=0
$$

where the $P_{i j}$ are rates of all processes that transfer an atom from a given state $i$ to a state $j$. For the calculation of these terms we account for radiative excitation and deexcitation, radiative ionization and recombination and corresponding collisional processes. We solve statistical equilibrium equations (3.3) for the elements listed in Table 1. Model atoms are taken mostly from TLUSTY (Hubeny 1988, Hubeny \& Lanz 1992, 1995). The set of model atoms is slightly larger for hot star wind modelling. For this purpose we used data from the Opacity Project (Seaton 1987) and from the Iron Project (Hummer et al. 1992). For an acceleration of the convergence of the system of statistical equilibrium equations together with the radiative transfer equations we apply the Newton-Raphson method for the Sobolev radiative transfer in lines and accelerated lambda iterations in continuum (or, in other words, approximate Newton-Raphson iterations based on Rybicki \& Hummer (1992)). 
Table 1. Atoms and their ionization stages included into the NLTE equations

\begin{tabular}{llll}
\hline H I-II & He I-III & C I-IV & N I-IV \\
O I-IV & Ne I-IV & Na I-III & Mg II-IV \\
Al I-V & Si II-V & S II-V & Ar III-IV \\
Ca II-IV & Fe II-V & Ni II-V & \\
\hline
\end{tabular}

\subsection{Hydrodynamic equations}

The set of hydrodynamic equations has the following form:

$$
\begin{gathered}
\frac{\mathrm{d}}{\mathrm{d} r}\left(r^{2} \rho v_{r}\right)=0 \quad \text { (the continuity equation), } \\
v_{r} \frac{\mathrm{d} v_{r}}{\mathrm{~d} r}=g^{\mathrm{rad}}-g-\frac{1}{\rho} \frac{\mathrm{d}}{\mathrm{d} r}\left(a^{2} \rho\right) \quad \text { (the equation of motion), } \\
\frac{3}{2} v_{r} \rho \frac{\mathrm{d} a^{2}}{\mathrm{~d} r}+\frac{a^{2} \rho}{r^{2}} \frac{\mathrm{d}}{\mathrm{d} r}\left(r^{2} v_{r}\right)=Q^{\mathrm{rad}} \quad \text { (the energy equation), }
\end{gathered}
$$

where $\rho$ is the wind density, $v_{r}$ is the radial velocity, $g$ is the acceleration of gravity, $a$ is the isothermal sound speed, $g^{\mathrm{rad}}=g_{\text {lines }}^{\mathrm{rad}}+g_{\mathrm{el}}^{\mathrm{rad}}$ is the radiative acceleration consisting of contributions from the lines $g_{\text {lines }}^{\mathrm{rad}}$ and from electron scattering $g_{\mathrm{el}}^{\mathrm{rad}}$, and $Q^{\mathrm{rad}}$ is the radiative heating/cooling term calculated using the thermal balance of electrons method (Kubát et al. 1999). Note that although the model solves multicomponent equations, the hydrodynamic equations above are written in their one-component forms since multicomponent effects can be neglected for A supergiants as a result of our calculations (except for helium decoupling, see Krtička \& Kubát 2005).

The radiative force in the Sobolev approximation is calculated as the sum of the contributions of individual lines after Castor (1974),

$$
g_{\mathrm{i}}^{\mathrm{rad}}=\frac{8 \pi}{\rho_{\mathrm{i}} c^{2}} \frac{v_{r}}{r} \sum_{\text {lines }} \nu H_{c} \int_{\mu_{c}}^{1} \mu\left(1+\sigma \mu^{2}\right)\left(1-e^{-\tau_{\mu}}\right) \mathrm{d} \mu .
$$

The line parameters used for the calculation of the radiative force are taken from the VALD database (Kupka et al. 1999). Occupation numbers determined in the course of the solution of the statistical equilibrium equations are then used for the radiative force calculation.

The hydrodynamic equations are solved using Newton-Raphson method. For more details we refer interested readers to Krtička (2003). To obtain faster model convergence, derivatives of occupation numbers with respect to fluid variables (i.e. $v_{r \mathrm{i}}, \rho_{\mathrm{p}}, \rho_{\mathrm{e}}$ and $T_{\mathrm{e}}$ ) are calculated from the statistical equilibrium equations (3.3). These derivatives are accounted for in the Newton-Raphson iteration step. This is similar to the implicit linearization of $b$-factors discussed in Kubát (1997).

\section{Stellar wind model of HD 12953}

To show the ability of our code to predict correct wind parameters of A supergiants we present a wind model of HD 12953. The parameters of this star are (after Kudritzki et al. 1999) $T_{\text {eff }}=9100 \mathrm{~K}, R=145 R_{\odot}$, and $M=9.7 M_{\odot}$. The wind mass-loss rate derived from observation $\dot{M}=4.3 \times 10^{-7} M_{\odot}$ year $^{-1}$ and the observed wind terminal velocity $v_{\infty}=150 \mathrm{~km} \mathrm{~s}^{-1}$ (Kudritzki et al. 1999) are in a good agreement with the calculated wind parameters $\dot{M}=1.3 \times 10^{-7} M_{\odot}$ year $^{-1}$ and $v_{\infty}=140 \mathrm{~km} \mathrm{~s}^{-1}$. The model radial velocity and the temperature structure are displayed in Figure 2. 

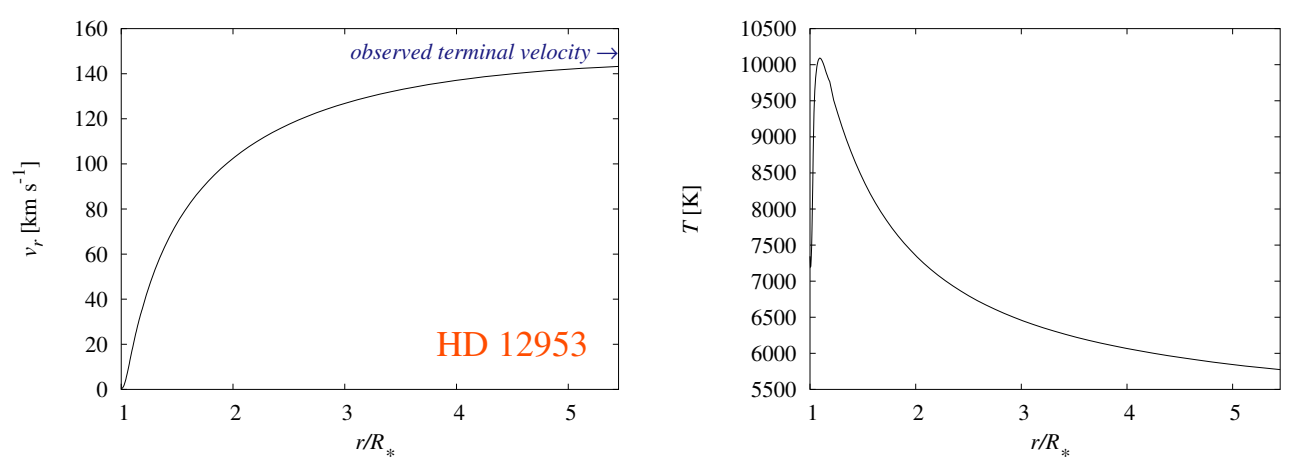

Figure 2. The calculated model of HD 12953. Left panel: Wind radial velocity. Right panel: Wind temperature structure.

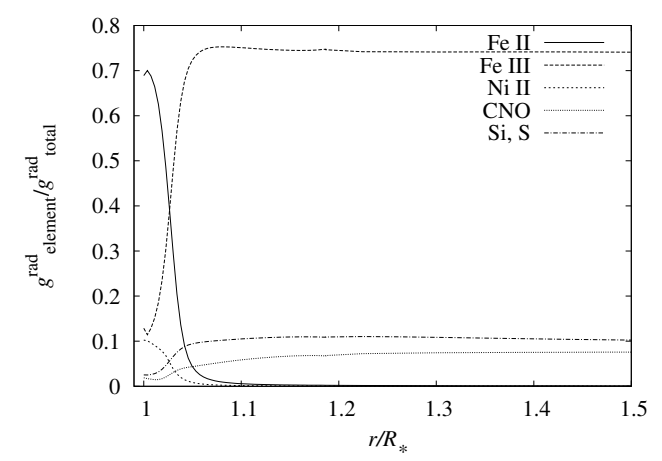

Figure 3. Relative contribution of individual atoms and their ionization states to the radiative acceleration

As can be seen from Figure 3, iron is the most important atom for the radiative acceleration. However, the contributions of the iron ionization states depend on the radius. While Fe II is important close to the star, Fe III is important mostly in the outer wind regions. The contributions of the light elements such as $\mathrm{C}, \mathrm{N}, \mathrm{O}, \mathrm{Si}$ and $\mathrm{S}$ are much smaller.

\section{Conclusions}

We have presented our NLTE wind models of A supergiants which are able to predict both detailed wind structure and wind parameters (i.e. mass-loss rates and terminal velocities). Although our models are still preliminary, they are able to predict correct wind parameters of A supergiants, as was demonstrated in the case of HD 12953. 


\section{Acknowledgements}

This work was supported by grants GA ČR 205/02/0445, 205/03/D020, 205/04/1267, MVTS SR-CR 128/04. JKr is grateful to IAU for travel grant. The Astronomical Institute Ondřejov is supported by projects K2043105 and Z1003909.

\section{References}

Anderson, L. S. 1989 ApJ 339, 558-578

Anderson, E., Bai, Z., Bischof, C., et al. 1999 LAPACK Users' Guide, 3rd ed., SIAM, Philadelphia

Castor, J. I. 1974 MNRAS 169, 279-306

Feautrier P. 1964 C. R. Acad. Sci. Paris 258, 3189

Gayley, K. G. \& Owocki, S. P. 1994 ApJ 434, 684-694

Hubeny, I. 1988 Comput. Phys. Commun. 52, 103

Hubeny, I. \& Lanz, T. 1992 A\&A 262, 501-514

Hubeny, I. \& Lanz, T. 1995 ApJ 439, 875-904

Korčáková, D., Kubát J. \& D., Krtička, J. 2005, These Proceedings, AP4

Krtička, J. 2003 In Stellar Atmosphere Modelling (eds. I. Hubeny, D. Mihalas \& K. Werner) ASP Conf. Ser., Vol. 288, 259-262

Krtička, J. \& Kubát, J. 2001 A $\& A$ 377, 175-191

Krtička, J. \& Kubát, J. 2004 A\&A 417, 1003-1016

Krtička, J. \& Kubát, J. 2005, These Proceedings, 201

Kubát, J. 1993 Ph.D. thesis, Astronomický ústav AV ČR, Ondřejov

Kubát, J. 1997, A\&A 326, 277-286

Kubát, J. 2003 In Stellar Atmosphere Modelling (eds. I. Hubeny, D. Mihalas \& K. Werner) ASP Conf. Ser., Vol. 288, 87-90

Kubát, J., Puls, J. \& Pauldrach, A. W. A. 1999 A $\& A$ 341, 587-594

Kudritzki, R. P., Puls, J., Lennon, D. J., Venn, K. A., Reetz, J., Najarro, F., McCarthy, J. K., Herrero, A. 1999 ApJ 350, 970-984

Kupka F., Piskunov, N. E., Ryabchikova, T .A., Stempels, H. C., \& Weiss, W. W. 1999 A\& AS 138, 119-133

Mihalas, D. \& Hummer, D. G. 1974 ApJS 28, 343-372

Pauldrach, A. W. A. 1987, A\& A 183, 295-313

Rybicki, G. B. \& Hummer, D. G. 1978 ApJ 219, 654-675

Rybicki, G. B. \& Hummer, D. G. 1992 A\& A 262, 209-215

Seaton, M. J. 1987 J. Phys. B 20, 6363-6378

\section{Discussion}

LANDSTREET: The radiative forces are exerted mainly on metal ions rather than on $\mathrm{H}$ and He. There is presumably some difference in the outflow speed with frictional coupling. Is frictional heating of the wind important?

KRTIČKA: The wind components are well coupled in these models. Thus frictional heating is not important in this case. However, for simplicity the helium decoupling was not allowed in these models (see Krtička \& Kubát 2005) for a discussion of helium decoupling.

PrZybilla: Can you comment on the match between observed P-Cygni lines and your predictions?

KRTIČKA: There is a poster (Korčáková et al. 2005) which discusses this issue. However, the results are not yet satisfactory. 